\title{
Microwave Characterization of Activated Carbons
}

\author{
Fatma Shkal ${ }^{*}$, Susana Garcia Lopez ${ }^{2}$, Daniel Slocombe' ${ }^{1}$, Adrian Porch ${ }^{1}$ \\ ${ }^{1}$ School of Engineering, Cardiff University, Cardiff, United Kingdom, Cardiff, UK \\ ${ }^{2}$ Engineering \& Physics Science School, Heriot-Watt University, Edinburgh, UK \\ Email: `ShkalFA@cardiff.ac.uk, S.Garcia@hw.ac.uk, SlocombeD1@cardiff.ac.uk, PorchA@cardiff.ac.uk
}

How to cite this paper: Shkal, F., Lopez, S.G., Slocombe, D. and Porch, A. (2018) Microwave Characterization of Activated Carbons. Journal of Computer and Communications, 6, 112-123. https://doi.org/10.4236/jcc.2018.61012

Received: August 7, 2017

Accepted: December 26, 2017

Published: December 29, 2017

\begin{abstract}
Microwave complex permittivity measurements of activated carbons were investigated using two different microwave measurement techniques, microwave cavity perturbation at $2.5 \mathrm{GHz}$ and broadband coaxial probe in the frequency range between $10 \mathrm{MHz}$ and $10 \mathrm{GHz}$. Such measurements demonstrate the ability of these materials to interact with the microwave electric field, both in terms of strong polarization and absorption. We have thus used complex permittivity to quantify the variability of different forms of activated carbon produced from different sources and different treatments, such as thermal treatments or impregnation. In addition, the cylindrical cavity at $2.5 \mathrm{GHz}$ was used for temperature-dependent dielectric properties measurements of activated carbons to investigate their susceptibility to microwave heating as function of temperature. Microwave complex permittivity of activated carbons was measured in the temperature range from $30^{\circ} \mathrm{C}$ up to $150^{\circ} \mathrm{C}$.
\end{abstract}

\section{Keywords}

Activated Carbon, Cavity Perturbation, Coaxial Probe, Dielectric Properties

\section{Introduction}

Activated carbon materials have many industrial applications, especially in purification of gases and water technologies, and many studies have attempted to characterize their different dielectric properties. In fact, most black carbon materials share the characteristic of strong microwave absorption, so they will heat as a result of their interaction with a strong electric field. This might be advantageous, for example, in the thermal regeneration of saturated activated carbons by microwaves [1] [2] [3].

The interaction of dielectric materials with a microwave electric field can be 
quantified by a complex relative permittivity $\varepsilon^{*}=\varepsilon^{\prime}-j \varepsilon^{\prime \prime}$, whose real part $\varepsilon^{\prime}$ indicates the stored electrical energy (or polarization) of the material, while the imaginary part $\varepsilon^{\prime \prime}$ indicated the power loss within the material [4].

\section{Theory}

Microwave measurements of materials are usually based around two common techniques, namely Transmission/Reflection techniques and Resonance techniques. The former are used for measurement of real part of permittivity of both high and low loss materials over a wide frequency range, based on measurement of the scattering parameters $S_{11}$ and $S_{21}$ of a dielectric-loaded probe or sample cell [5] [6] [7].

Resonance techniques typically have the utility for measuring low loss materials, but only at spot frequencies allowed by the host resonator. They are also selected for characterization of lossy liquids because of the flexibility over the specific size and geometry of samples, added to their high inherent accuracy [8].

Cavity-type resonators are superior to lumped element resonators for materials measurement since their quality factor $(\mathrm{Q})$ can reach tens of thousands, typically an order of magnitude higher than for lumped element resonators.

For microwave dielectric property measurements using microwave cavities, cavity perturbation analysis is used to calculate the complex permittivity by measurements of the resonant frequency shift and the change of quality factor on inserting the sample in the cavity resonator, with high $\mathrm{Q}$ for the host cavity desirable for accurate results [5] [9] [10].

In this study, we used cylindrical, aluminum cavity resonator of inner diameter $92 \mathrm{~mm}$ and inner length $40 \mathrm{~mm}$ operating in its $\mathrm{TM}_{010}$ mode at $2.5 \mathrm{GHz}$, where there is high electric field on the axis, where the sample is placed. We also used a broadband coaxial probe to investigate the dielectric properties of different forms of activated carbons, in the frequency range between $10 \mathrm{MHz}$ and 10 GHz.

\subsection{Broadband Coaxial Probe Method}

The coaxial probe technique is used to measure the broadband dielectric properties of materials. The complex permittivity of the samples can be extracted using a capacitive model which is shown in Figure 1 . The complex permittivity can be calculated from measured reflected coefficient $S_{11}$ from the reflected signal.

$$
s_{11}^{*}=s_{11} e^{j \theta}=\frac{1-Y_{s}}{1+Y_{s}} e^{j \theta}=\frac{1+j \omega z_{0}\left(\varepsilon_{r} c_{0}+c_{f}\right)}{1-j \omega z_{0}\left(\varepsilon_{r} c_{0}+c_{f}\right)}
$$

where $Z_{0}$ is the characteristic impedance of the coaxial line (here $50 \Omega$ ), $C_{0}$ is the capacitance of the probe terminated in free space, $C_{f}$ is the fringing capacitance, $\omega$ is the angular frequency, $\theta$ is the phase associated with the electrical length of the coaxial probe and $\varepsilon$ is the complex permittivity of the sample. The complex relative permittivity can be derived using. 


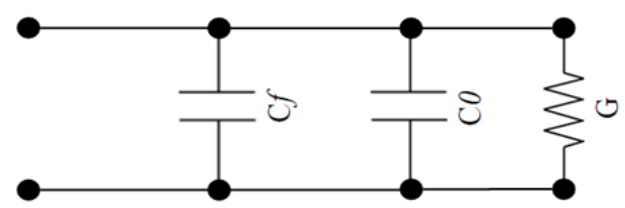

Figure 1. Equivalent circuit of an open-ended coaxial probe.

$$
\varepsilon_{r}=\frac{1-s_{11}^{*}}{j \omega z_{0} c_{0}\left(1+s_{11}^{*}\right)} \frac{c_{f}}{c_{0}}
$$

The two unknown capacitances can be calculated by measuring material with known dielectric permittivity such as water [11] [12] [13] [14].

\subsection{Cavity Perturbation Method}

Cavity perturbations is a means of measuring material properties by analysis of the changes in resonant frequency and $\mathrm{Q}$ factor when a dielectric material sample is inserted into the cavity. For the $\mathrm{TM}_{010}$ mode, the perturbation calculations of the real and imaginary parts of the complex permittivity can be simply derived using two approximate formulas below:

$$
\begin{aligned}
& \varepsilon_{r}^{\prime \prime} \approx 2 J_{1}^{2}\left(p_{01}\right) \frac{f_{0}-f_{s}}{f_{0}} \frac{R_{c}^{2}}{R_{s}^{2}} \\
& \varepsilon_{r}^{\prime \prime} \approx J_{1}^{2}\left(p_{01}\right)\left(\frac{1}{Q_{s}}-\frac{1}{Q_{0}}\right) \frac{R_{c}^{2}}{R_{s}^{2}}
\end{aligned}
$$

where $f$ is the resonant frequency, $Q$ is the quality factor and the subscripts s and 0 refer to the sample loaded and empty cavities, respectively; $R_{c}$ is the inner radius of the cavity, $R_{s}$ is the radius of the cylindrical sample tube (assuming that it occupied the full inner length of the cavity, on-axis), $J_{1}$ is the first order Bessel function of the first kind and $p_{01}=2.405$, so that $J_{1}\left(p_{01}\right)=0.52$ [15] [16] [17].

\subsection{Temperature-Dependent Dielectric Measurements}

Change in the ambient temperature of the cavity will cause change in the resonant frequency, due to change in thermal expansion of the cavity which cause change in the cavity dimensions, radius (a) and length (I). The thermal expansion coefficient $\alpha$ of the aluminum cavity can be used to describe these changes. Therefore, the assumption of that the volume of the cavity is fixed is not valid in the case of changing the temperature. The perturbation Equations (3) and (4) are valid in the case of the sample size is small compared to the cavity size. Therefore, any change in the sample size due to temperature variation must be included in the calculations.

In this work, the nodal modes were used for temperature correction measurements. Nodal cavity modes are those which have no electric fields in the center of frequency and are therefore unaffected by the presence of the sample. Figure 2 shows the electric and magnetic distributions of some nodal modes for the cavity with $40 \mathrm{~mm}$ radius and $46 \mathrm{~mm}$ height. 

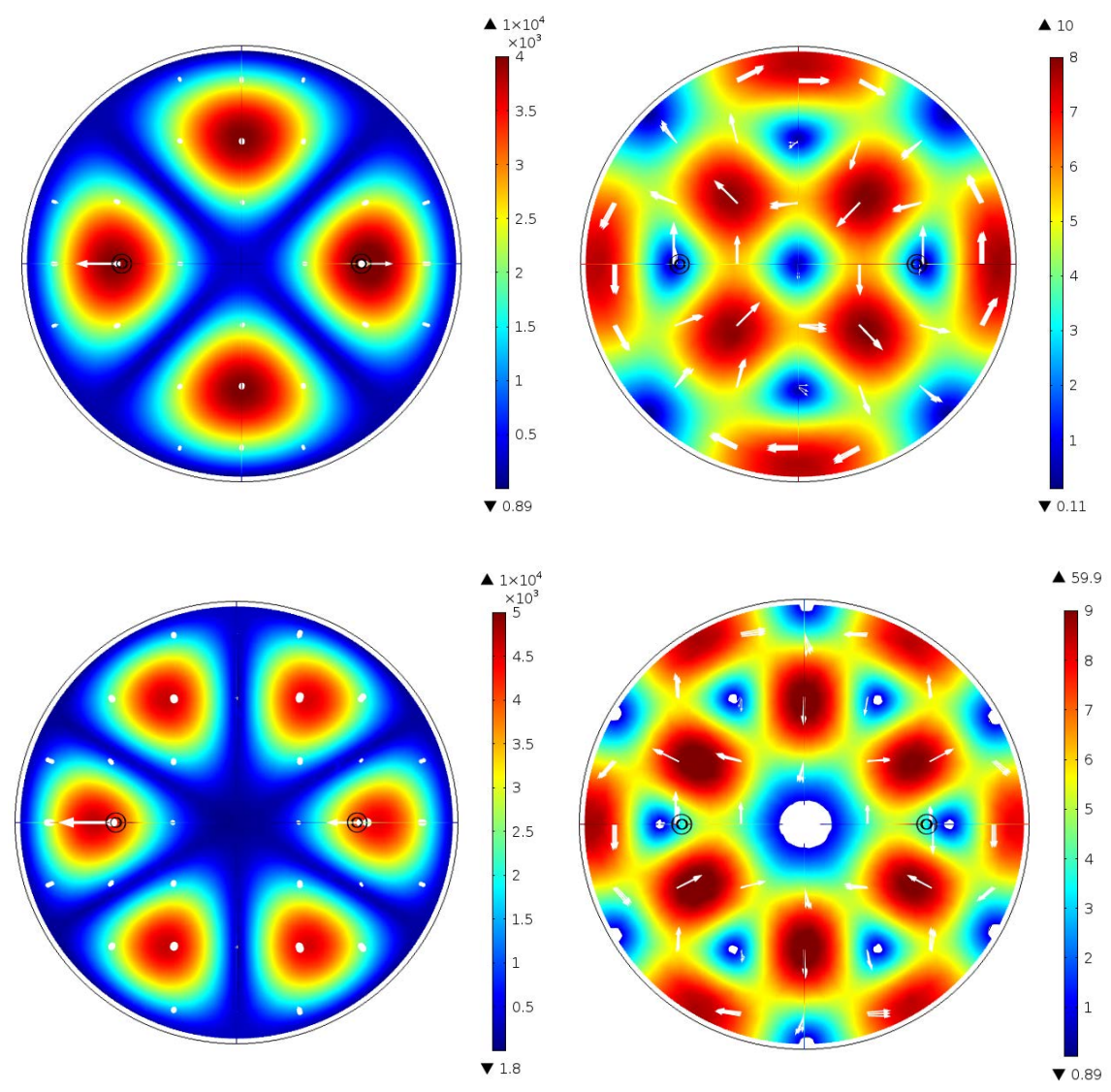

Figure 2. Field distributions of electric (left) and magnetic (right) fields of nodal cavity modes $\mathrm{TM}_{210}$ (top) and $\mathrm{TM}_{310}$ (bottom).

The simplest way to obtain the temperature correction is to subtract the fractional frequency shift of nodal modes such as $\mathrm{TM}_{310}$ (of frequency $6.61 \mathrm{GHz}$ ) from the fractional shift of the measurement mode $\mathrm{TM}_{010}$ [18] [19] [20].Through cavity perturbation, the change in permittivity with respect to a reference temperature can then be expressed as following:

$$
\begin{gathered}
\frac{\Delta f_{s}(T)}{f_{s}(0)}=\left[\frac{\Delta f_{010, s}(T)}{f_{010, s}(0)}-\frac{\Delta f_{m 10, s}(T)}{f_{m 10, s}(0)}\right]-\left[\frac{\Delta f_{010, u}(T)}{f_{010, u}(0)}-\frac{\Delta f_{m 10, u}(T)}{f_{m 10, u}(0)}\right] \\
\varepsilon_{1}(T) \approx-2\left[\frac{f_{s}(T)-f_{0}(0)}{f_{0}(0)}\right] \frac{v_{c}}{v_{s}} G_{m m p}+1 \\
\varepsilon_{2}(T)=\left[\frac{1}{Q_{s}(T)}-\frac{1}{Q_{0}(0)}\right] \frac{v_{c}}{v_{s}} G_{n m p}
\end{gathered}
$$

\section{Experiments}

\subsection{Samples}

The samples measured in these experiments and their description are listed in Table 1. The samples were formed from different particle sizes. They were crushed using a mortar and pestle, and then sieved by $63 \mu \mathrm{m}$ Lab sieve to be approximately $60 \mu \mathrm{m}$ size, as illustrated schematically in Figure 3. 


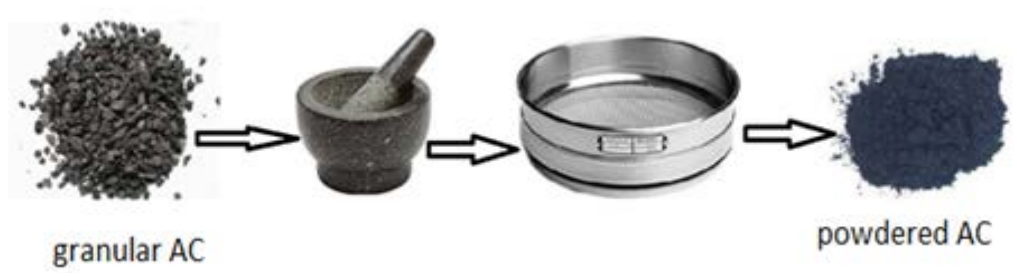

Figure 3. Grinding the granular activated carbon to powder.

Table 1. Activated carbon samples.

\begin{tabular}{ccl}
\hline Material & Label & \multicolumn{1}{c}{ Description } \\
\hline Norit GCN 3070 & AC1 & Coconut shell based granular AC \\
MW & AC2 & Norit after 25 Microwave (MW) heating cycle \\
CH & AC3 & Norit after 25 Conventional heating $(\mathrm{CH})$ cycle, using a heating tape \\
GKOS & AC4 & Ground olive stone derived AC \\
GKOS-N800 & AC5 & GKOS with 800 ${ }^{\circ}$ C treated NH3 impregnation \\
Calgon BLP & AC6 & Bituminous coal based GAC \\
\hline
\end{tabular}

\subsection{Dielectric Properties of Activated Carbon at $2.498 \mathrm{GHz}$}

The samples were filled in quartz tubes, with inner and outer diameters of $2 \mathrm{~mm}$ and $2.4 \mathrm{~mm}$, respectively. The sample tube was centered in the cavity using cylindrical placement ring, as shown in Figure 4. The measurements were repeated at least 10 times for each sample; so that mean values (and standard deviation of the mean) can be estimated reliably for each sample.

\subsection{Broadband Coaxial Probe Measurements}

The measurements was carried out using coaxial flat probe with a square flange which was connected to an Agilent PNA-L vector network analyzer, after careful calibration was done using an Agilent $85052 \mathrm{D}$ calibration Kit; the set-up is shown in Figure 5.

The samples were prepared as mixtures of silicone rubber purchased from $\mathrm{MB}$ Fiberglass, Northern Ireland, with different concentrations of powdered activated carbon (AC1) to avoid the uncertainty resulting from the contacts between the powder particles, . Fifteen samples with three different contents of activated carbon were prepared at ratios of mass at 2:1, 4:1 and 8:1. The preparation of samples started by mixing the two parts of silicone and hardener, then the powder was added to the mixture, which was left from $6-8$ hours at $25^{\circ} \mathrm{C}$ to cure.

Pure silicone rubber samples were also measured to investigate the errors resulting from the air bubbles, which were observe to appear as part of the hardening process.

\subsection{Temperature Dependent Properties of Activated Carbon}

The temperature dependent properties of activated carbon were measured using 


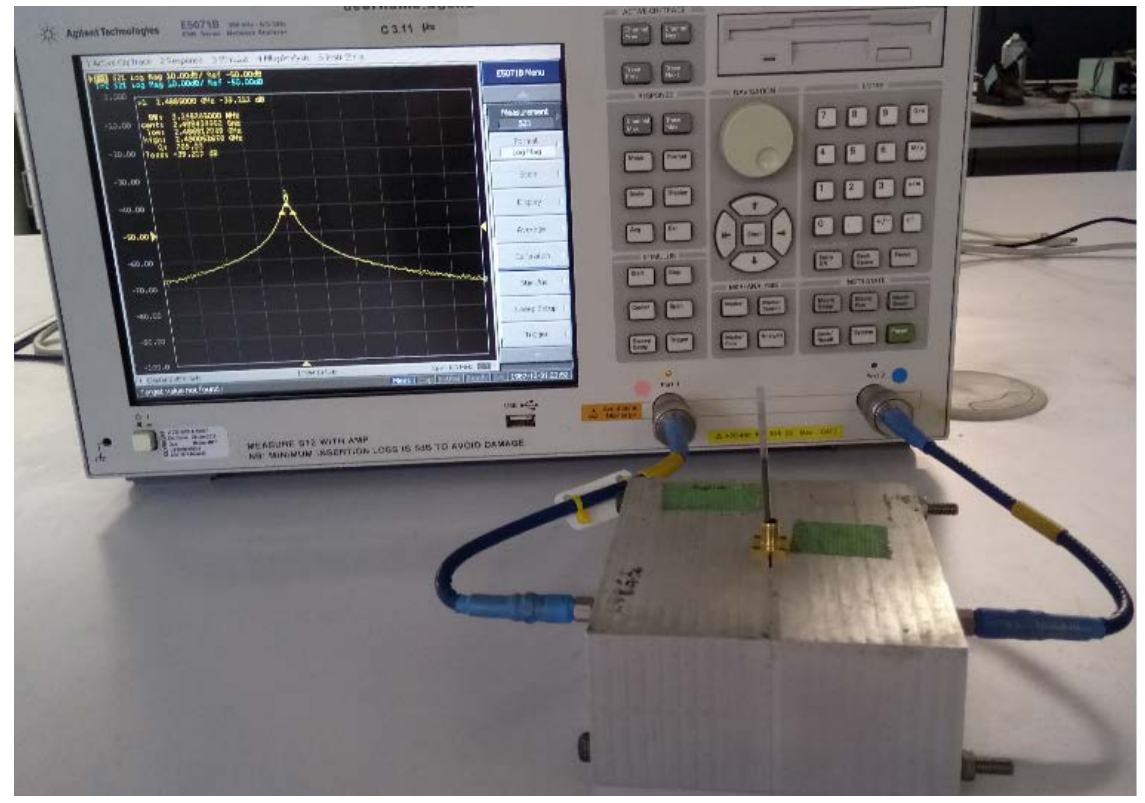

Figure 4. Photograph of the experiment set-up for cavity measurements.

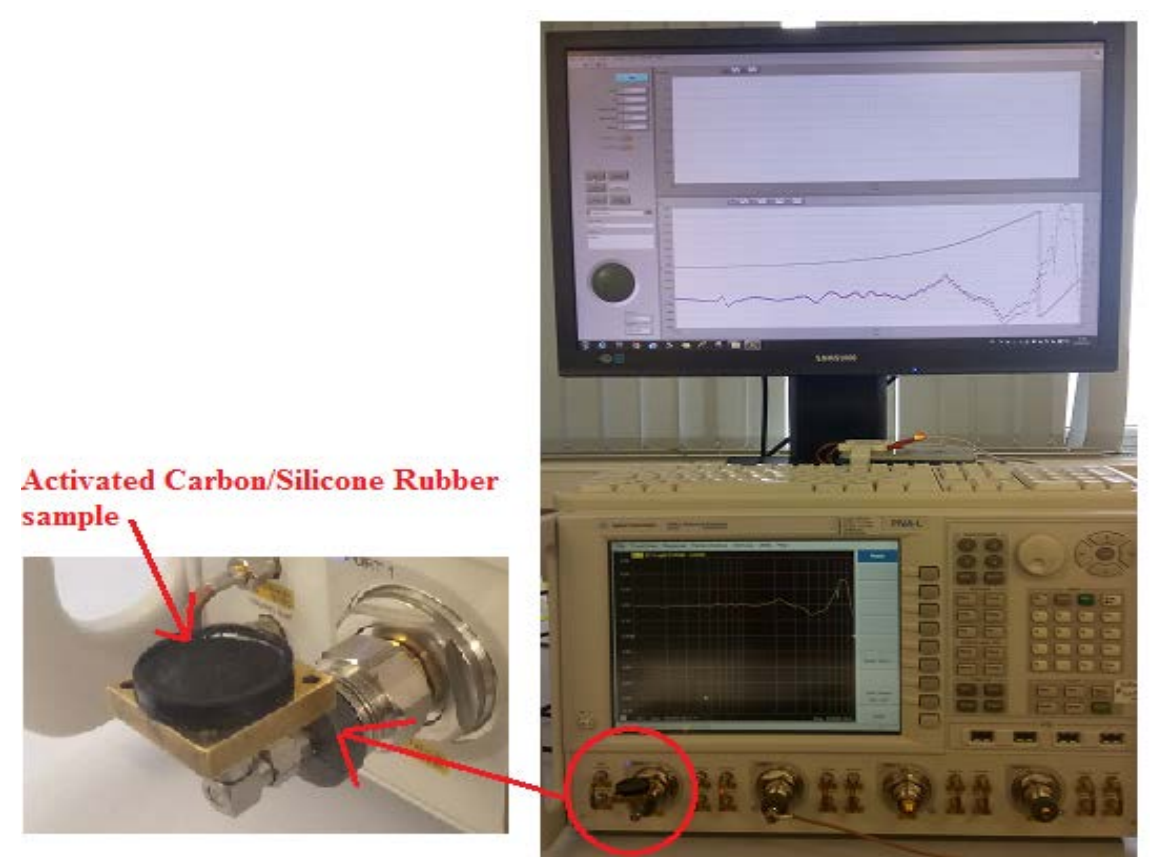

Figure 5. Photograph of coaxial probe experimental setup. The coaxial flat probe is connected to an Agilent PNA-L N5232A network analyzer under LabVIEW program control.

the microwave cavity technique at a frequency of $2.5 \mathrm{GH}$ in the temperature range $30^{\circ} \mathrm{C}$ to $150^{\circ} \mathrm{C}$.

The cavity loaded sample was placed inside a Memmert UF30 laboratory oven, as shown in Figure 6. The change in the resonant frequency and bandwidth were recorded using a custom LabVIEW program. Temperature was measured using resistance temperature detector PT100 RTDs provided by Omega, interfaced to a National Instruments C-DAQ. At each temperature, the 


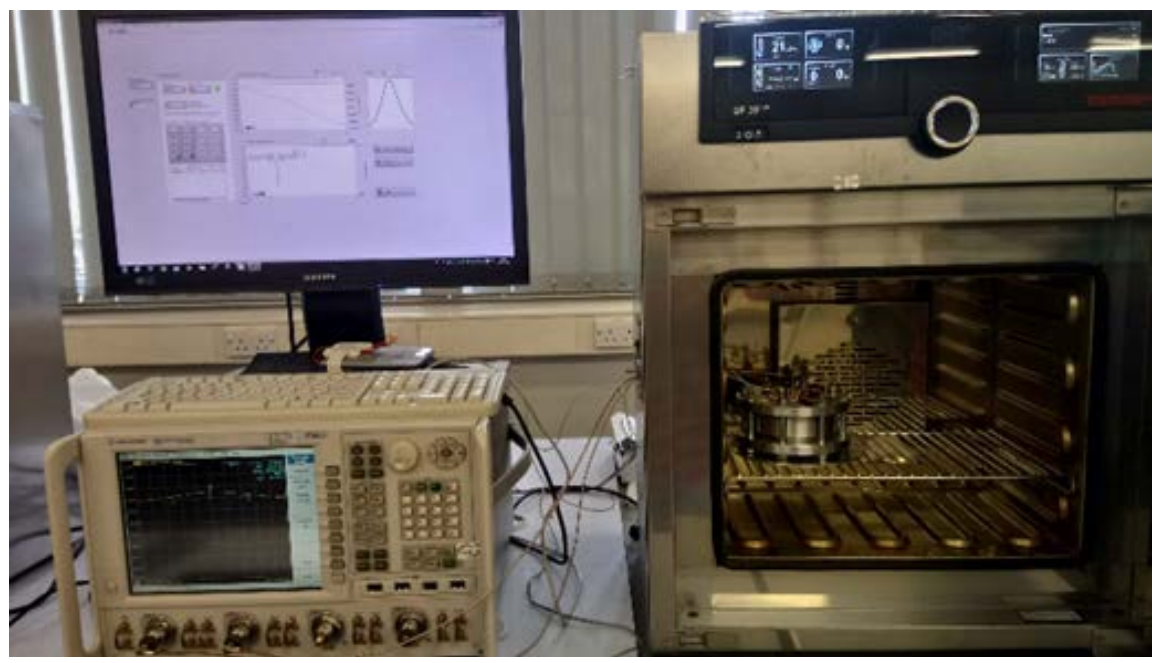

Figure 6. Photograph the bench-top experimental assembly for cavity measurements. The cavity is put inside a computer-controlled oven and connected to a network analyzer under LabVIEW program control.

magnitude of the transmission parameter $\mathrm{S}_{21}$ was measured with the PNA-L network analyzer N5232A to obtain the resonant frequency and quality factor $\mathrm{Q}$ of the resonator

\section{Results}

\subsection{Dielectric Properties of Activated Carbon at $2.498 \mathrm{GH}$}

The real and imaginary parts of the complex permittivity are shown in Figure 7 and Figure 8, which illustrate the average values of the measurements of complex permittivity for all samples. Figure 7 and Figure 8 show that the dielectric constant and loss factor of the powdered samples for all activated carbon samples are significantly different. The small errors are associated with the small differences in sample volumes each time a powder sample is loaded into the cavity (since they are powders, with random packing nature, and all tubes have a volume size distribution giving an error of about $2 \%$ ).

\subsection{Coaxial Probe Measurements}

The dielectric constant $\mathcal{\varepsilon}^{\prime}$ and loss factor $\varepsilon^{\prime \prime}$ measurements of activated carbon samples were performed on samples which were mixed with silicone rubber in three different ratios of mass at 2:1, 4:1 and 8:1. Five samples of each ratio were prepared at different times with separate mixtures, to allow for standard errors to be assessed, and each sample was measured at least 10 times. Figure 9 and Figure 10 show the dielectric constant and loss factor of silicone rubber/activated carbon blends as function of frequency. In the lower frequency region the dielectric constant fluctuated up to $100 \mathrm{MHz}$. However, in the higher frequency region the dielectric constant values remained approximately constant up to about $5 \mathrm{GHz}$. The dielectric constant and loss factor change according to the content of activated carbon in the mixture. It has been found that the 


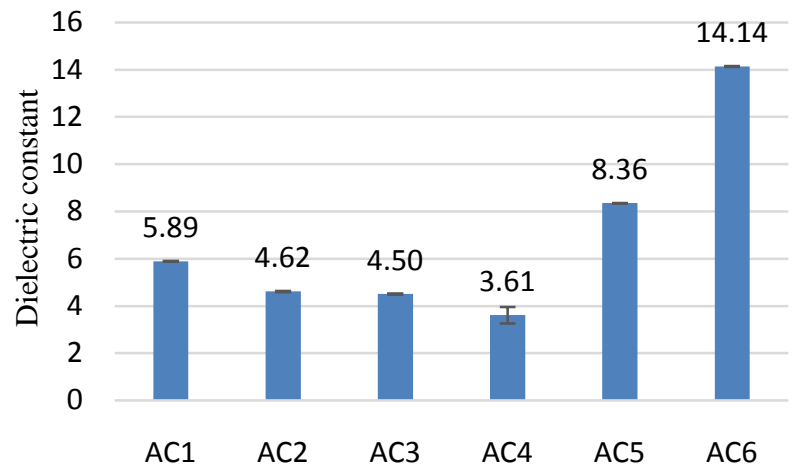

Figure 7. Dielectric constant $\mathcal{\varepsilon}$ for activated carbon samples.

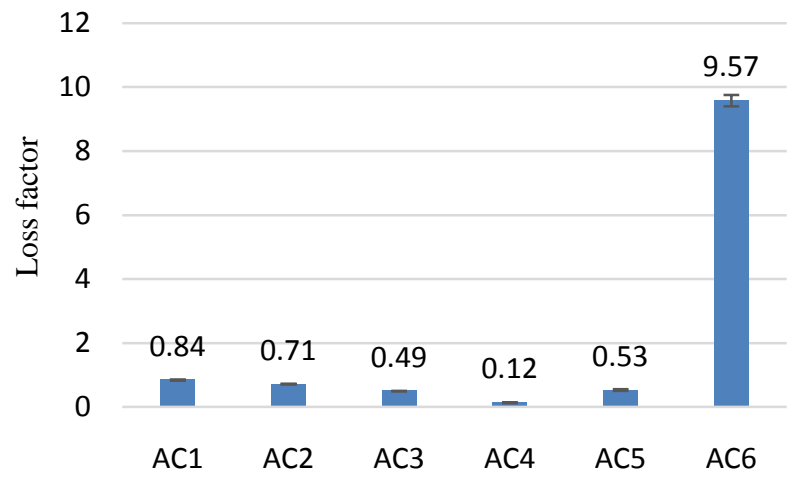

Figure 8. Loss factor $\varepsilon^{\prime \prime}$ for activated carbon samples.

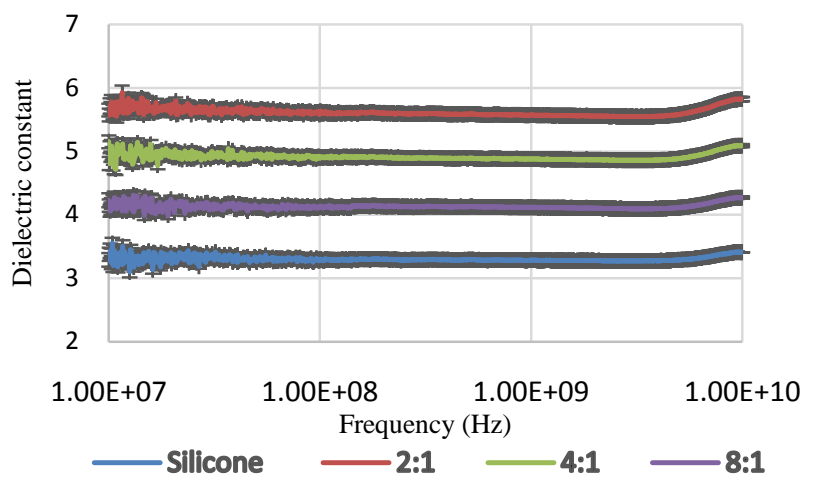

Figure 9. Dielectric constant for silicone rubber/AC1 samples.

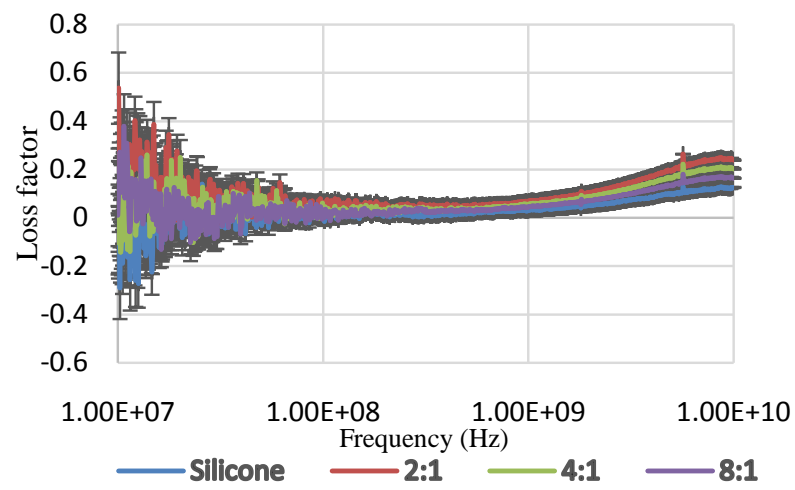

Figure 10. Loss factor for silicone rubber/AC1 samples. 
increasing filler content of activated carbon leads to increases in both the relative permittivity and dielectric loss factor. This is owing to effective values of complex permittivity being extracted, which is a function of volume fraction. We have not attempted to extract the intrinsic permittivity of the samples using effective medium theory but we would expect these to be approximately the same, irrespective of the concentration. We explain the great variation in loss factor below around $100 \mathrm{MHz}$ as being due to the variations in the contacts between the particles. This gives way to a loss peak at higher frequencies, centered at around $10 \mathrm{GHz}$ and indicative of the intra-particle electrical conductivity of the material.

\subsection{Temperature Dependent Properties of Activated Carbons}

Figures 11-14 show the change in the dielectric properties of two samples of activated carbon, labelled as $\mathrm{AC} 1$ and $\mathrm{AC} 4$, at $2.5 \mathrm{GHz}$ and as a function of temperature. The dielectric constant of both samples decreases linearly with temperature in the range between room temperature and about $120^{\circ} \mathrm{C}$, after which there are increases which might due to the release of the water vapor or other agents which adsorbed on the surface of activated carbons, although this is not consistent with the exact temperature of the onset these changes; furthermore, a

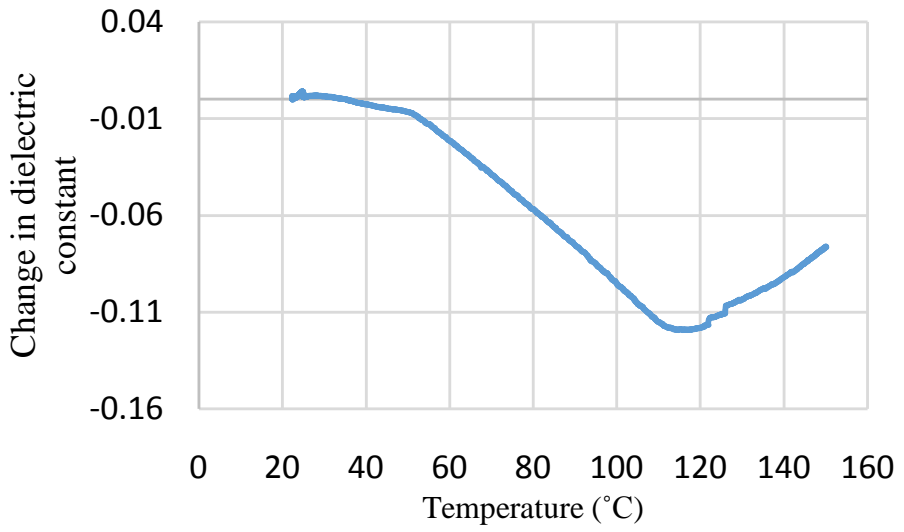

Figure 11. Change in dielectric constant of $\mathrm{AC} 1$ versus temperature.

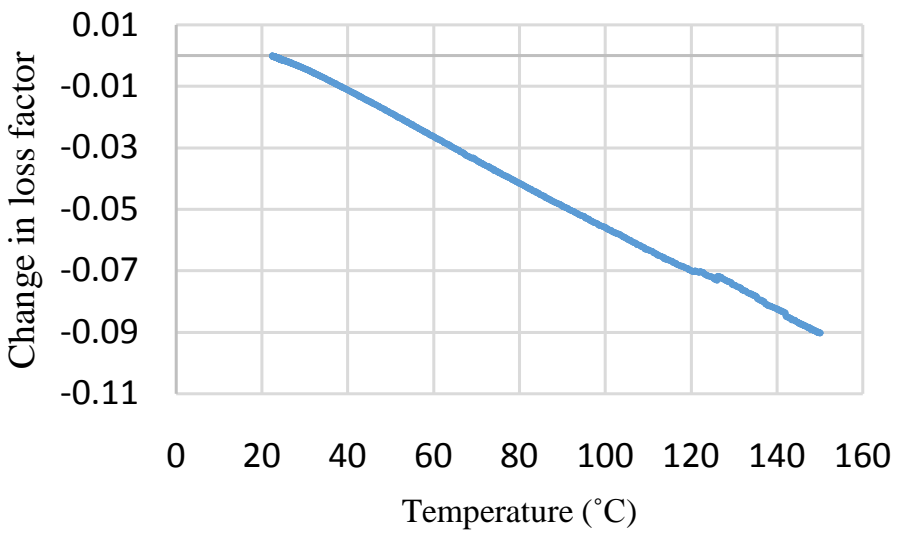

Figure 12. Change in loss factor of $\mathrm{AC} 1$ versus temperature. 


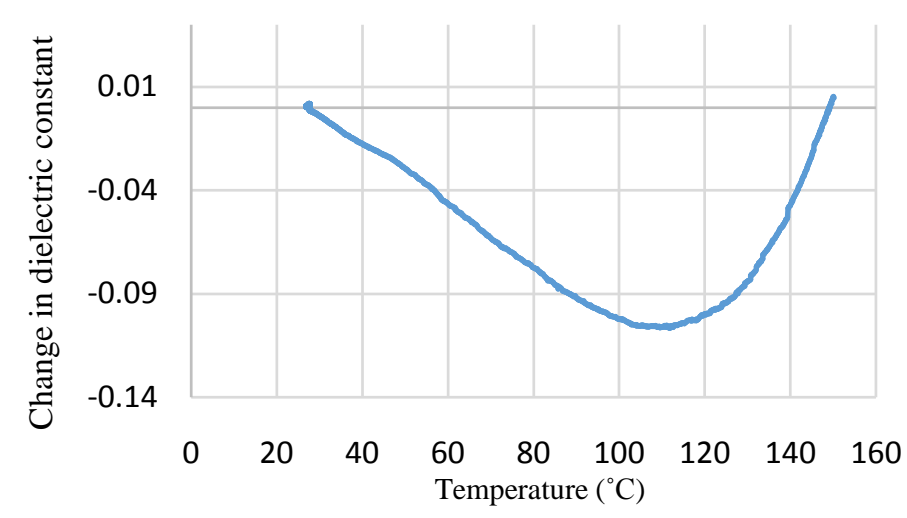

Figure 13. Change in dielectric constant of AC4 versus temperature.

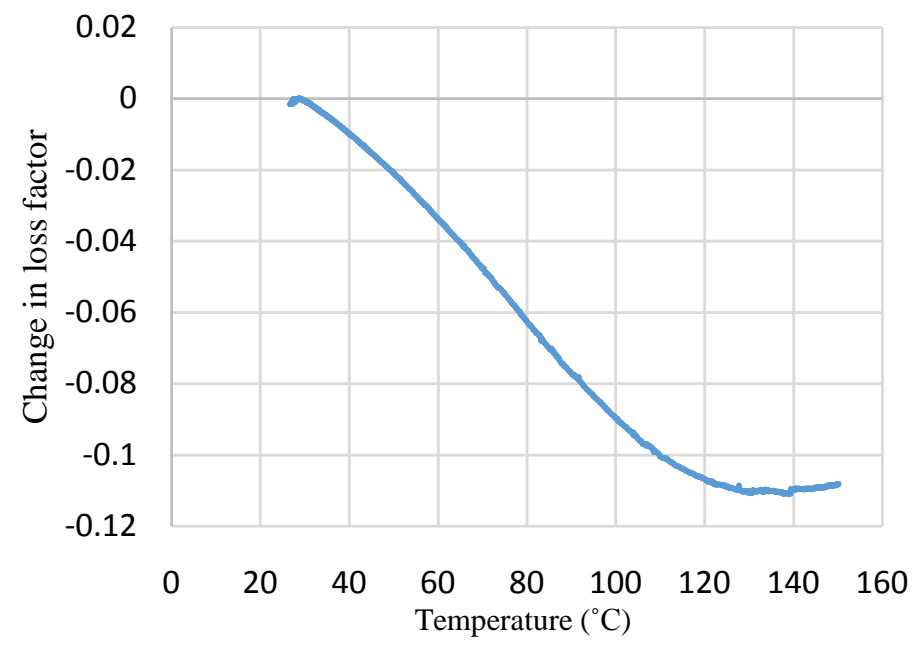

Figure 14. Change in loss factor of AC4 versus temperature.

loss in water should cause a further decrease of permittivity, owing to its highly polar nature, and this is not what is observed in the experimental data. The origin of the change is currently unknown and will be investigated by other techniques (e.g. downstream mass spectrometry).

\section{Conclusion}

In conclusion, we have investigated the complex permittivity of activated carbon samples at a frequency of $2.5 \mathrm{GHz}$ using cavity perturbations. The results show that the dielectric properties of activated carbons change depending on the raw materials that have been used in their production; for example, AC6, which was produced from Bituminous coal, has the highest dielectric constant and loss factor, at 14.1 and 9.6 respectively. It is also noticed that the both AC2 and AC3 samples, which were pre- heated using conventional heating and microwave heating, respectively, have approximately the same complex permittivities. Moreover, we can conclude that all activated carbon samples can be sufficiently heated by microwaves when exposed to high microwave electric field values, as their dielectric loss factor are high enough. The microwave dielectric properties 
of activated carbon/ silicone rubber mixtures were investigated at $10 \mathrm{MHz}$ to 10 $\mathrm{GHz}$ using a coaxial probe, with increasing content of activated carbon within a silicone host. The results showed increases in dielectric constant and dielectric loss with increasing mass fraction of activated carbon in the sample. In addition, the temperature dependent dielectric properties of some samples have been measured in the temperature range from $30^{\circ} \mathrm{C}$ up to $150^{\circ} \mathrm{C}$. The measurements were made at $2.5 \mathrm{GHz}$ with a $\mathrm{TM}_{010}$ cylindrical cavity resonator placed inside a laboratory oven. Measurements show that the dielectric constant varies with temperature depending on the nature of the materials. As all the samples have the ability to strongly adsorb vapor of water or/and another agents, so it is expected that heating them will vary their dielectric constant; however, the changes observed for these samples on heating is not consistent with the loss of water above $100^{\circ} \mathrm{C}$.

\section{Acknowledgements}

The first author gratefully acknowledges the support provided by the Libyan Embassy through the Cultural Libyan Attaché during her PhD project.

\section{References}

[1] Atwater, J.E. and Wheeler, R.R. (2003) Complex Permittivities and Dielectric Relaxation of Granular Activated Carbons at Microwave Frequencies between 0.2 and 26 GHz. Carbon, 41, 1801-1807. https://doi.org/10.1016/S0008-6223(03)00150-7

[2] Atwater, J. and Wheeler Jr., R. (2004) Microwave Permittivity and Dielectric Relaxation of a High Surface Area Activated Carbon. Applied Physics A, 79, 125-129. https://doi.org/10.1007/s00339-003-2329-8

[3] Zaini, M.A.A., et al. (2015) Composition-and Temperature-Dependent of Dielectric Properties of Zinc Chloride-Palm Kernel Shell Mixture at Microwave Frequencies. International Journal of Materials Science and Engineering, 3, 301-309.

[4] Chen, L.-F., et al. (2004) Microwave Electronics: Measurement and Materials Characterization. John Wiley \& Sons Ltd., Chichester. https://doi.org/10.1002/0470020466

[5] Sheen, J. (2009) Comparisons of Microwave Dielectric Property Measurements by Transmission/Reflection Techniques and Resonance Techniques. Measurement Science and Technology, 20, Article ID: 042001. https://doi.org/10.1088/0957-0233/20/4/042001

[6] Krupka, J. (2006) Frequency Domain Complex Permittivity Measurements at Microwave Frequencies. Measurement Science and Technology, 17, R55-R70. https://doi.org/10.1088/0957-0233/17/6/R01

[7] Singh, D.P., et al. (2011) Microwave Cavity Technique to Study the Dielectric Response in 4-n-Heptyl-4-Biphenyl Nematic Liquid Crystal at $20.900 \mathrm{GHz}$ and 29.867 GHz. Measurement, 44, 605-610. https://doi.org/10.1016/j.measurement.2010.11.020

[8] Sklavounos, A.H. and Barker, N.S. (2014) Liquid-Permittivity Measurements Using a Rigorously Modeled Overmoded Cavity Resonator. IEEE Transactions on Microwave Theory and Techniques, 62, 1363-1372.

https://doi.org/10.1109/TMTT.2014.2321348 
[9] Che, W., et al. (2008) Permittivity Measurement of Biological Materials with Improved Microwave Cavity Perturbation Technique. 38th IEEE European Microwave Conference, Amsterdam, 27-31 October 2008, 905-908.

[10] Slocombe, D., Porch, A., Bustarret, E. and Williams, O.A. (2013) Microwave Properties of Nanodiamond Particles. Appl. Phys. Lett., 102, Article ID: 244102. https://doi.org/10.1063/1.4809823

[11] Cuenca, J.A., Thomas, E., Mandal, S., Williams, O. and Porch, A. (2015) Investigating the Broadband Microwave Absorption of Nanodiamond Impurities. IEEE-TMTTT, 63, 4110-4118. https://doi.org/10.1109/TMTT.2015.2495156

[12] Porch, A., et al. (2012) Microwave Treatment in Oil Refining. Applied Petrochemical Research, 2, 37-44. https://doi.org/10.1007/s13203-012-0016-4

[13] Stuchly, M.A. and Stuchly, S.S. (1982) Equivalent Circuit of an Open-Ended Coaxial Line in a Lossy Dielectric. IEEE Transactions on instrumentation and Measurement, 1001, 116-119. https://doi.org/10.1109/TIM.1982.6312533

[14] Gabriel, C., Chan, T. and Grant, E. (1994) Admittance Models for Open Ended Coaxial Probes and Their Place in Dielectric Spectroscopy. Physics in Medicine and Biology, 39, 2183-2200. https://doi.org/10.1088/0031-9155/39/12/004

[15] Li, X. and Jiang, Y. (2010) Design of a Cylindrical Cavity Resonator for Measurements of Electrical Properties of Dielectric Materials. Master Thesis, Gavle University, Gavle.

[16] Li, S., Akyel, C. and Bosisio, R.G. (1981) Precise Calculations and Measurements on the Complex Dielectric Constant of Lossy Materials Using TM/sub 010/Cavity Perturbation Techniques. IEEE Transactions on Microwave Theory and Techniques, 29, 1041-1048. https://doi.org/10.1109/TMTT.1981.1130496

[17] Riddle, B., Baker-Jarvis, J. and Krupka, J. (2003) Complex Permittivity Measurements of Common Plastics over Variable Temperatures. IEEE Transactions on Microwave Theory and Techniques, 51, 727-733. https://doi.org/10.1109/TMTT.2003.808730

[18] Cuenca, J.A. (2015) Characterization of Powders Using Microwave Cavity Perturbation. Ph.D. Thesis, Cardiff University, United Kingdom.

[19] Slocombe, D. (2012) The Electrical Properties of Transparent Conducting Oxide Composites. Ph.D. Thesis, Cardiff University, United Kingdom.

[20] Cuenca, Je.A., Slocombe, D.R. and Porch, A. (2017) Temperature Correction for Cylindrical Cavity Perturbation Measurements. IEEE Transactions on Microwave Theory and Techniques, 65, 2153-2161.

https://doi.org/10.1109/TMTT.2017.2652462 